\title{
The mixed truth about frequency effects on free recall: Effects of study list composition
}

\author{
Jason D. OZubko and Steve Joordens \\ University of Toronto, Scarborough, Ontario, Canada
}

\begin{abstract}
The mixed-list paradox is the finding that high-frequency words show a recall advantage in blocked lists, but that this advantage is reversed or nullified in mixed lists. We argue that this paradox has been poorly defined. Specifically, researchers should investigate random and alternating lists separately. We examine blocked, random, and alternating lists in our experiment. We hypothesize that a low-frequency recall advantage could arise due to asymmetrically strong links between low- and high-frequency words. Supporting our hypothesis, a lowfrequency recall advantage is observed in the random list, as is an asymmetry of association. In the alternating list, no recall advantage is observed, and neither is any asymmetry of association. Our results offer a clear challenge to future research to (1) consider the associations between word frequencies and (2) consider random and alternating lists separately.
\end{abstract}

Previous investigations of free recall have noted that in pure or blocked lists high-frequency words show a recall advantage. In randomly mixed or alternating lists however, either low-frequency words show an advantage or no advantage is observed. This phenomenon is sometimes referred to as the mixed-list paradox (Watkins, LeCompte, \& Kim, 2000). To examine the consistency of this effect, we gathered the results from free-recall studies by Brown, Conover, Flores, and Goodman (1991), DeLosh and McDaniel (1996), Gregg, Montgomery, and Castano (1980), Hicks, Marsh, and Cook (2005), May, Cuddy, and Norton (1979), Van Overschelde (2002), Ward, Woodward, Stevens, and Stinson (2003), and Watkins et al. (2000). For each set of results advantage values were calculated by subtracting the proportion of low-frequency words recalled from the proportion of high-frequency words recalled. A positive advantage value indicates a high-frequency recall advantage in an experiment whereas a negative advantage value indicates a low-frequency recall advantage was observed (zero indicates equal levels of recall). These advantage values were plotted separately for pure or blocked study lists versus random or alternating study lists as presented in Figure 1.

A $t$ test revealed that the average advantage value in pure or blocked study lists is significantly higher than the average advantage value in random or alternating lists $[t(21)=4.85, p<.01]$. Thus, it is clear that there is a general tendency for high-frequency words to show an advantage in pure lists but for that advantage to be eliminated or, more often, reversed in mixed lists.

One problem in explaining the mixed-list paradox is its poor definition. "Mixed-list" can be taken to mean either random (e.g., Van Overschelde, 2002; Ward et al., 2003) or alternating (e.g., Watkins et al., 2000), and the suggested "paradox" is described as either equal recall for high- and low-frequency words or a low-frequency recall advantage. The approach we take here is to explicitly investigate both alternating and random lists separately, with the goal of developing models and theories that make predictions about each kind of list.

One primary focus will be on how associations may develop between items during study, and how these associations contribute to a low-frequency recall advantage under certain conditions. We will operate under the assumption that, since more high than low-frequency words are recalled in pure lists, all else being equal, highfrequency words should show a recall advantage. Thus, for low-frequency words to show an advantage in mixed lists, strong associations in favor of low-frequency recall must be acting in opposition to the processes that would otherwise lead to a high frequency advantage.

Kahana (1996) provides a strong demonstration of the importance of association by contiguity by reexamining the recall data from several large scale recall studies (i.e., Murdock, 1962; Murdock \& Metcalfe, 1978; Murdock \& Okada, 1970). Kahana examined the conditional response probability of recalling word $x$, given one had just recalled word $y$. He concluded that the probability of recalling a word that was presented adjacent to the item just recalled was about three times higher than the probability of recalling some other word. In addition, the probability of adjacent forward recalls was about twice as likely as adjacent backward recalls. Thus, when recalling words based on a cue, there was a strong tendency for participants to recall words studied near the cue.

Association by contiguity is informative about the way in which participants generally recall words. However, if

J. D. Ozubko, jdozubko@uwaterloo.ca 


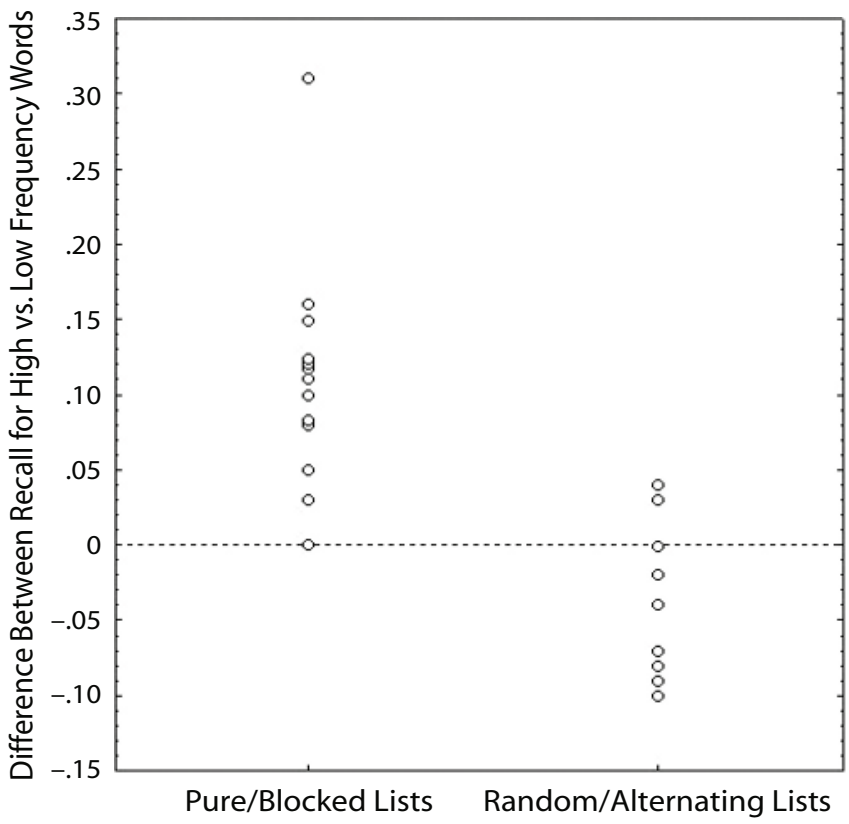

Figure 1.Advantage values from experiments from several freerecall studies (i.e., Brown et al., 1991; DeLosh \& McDaniel, 1996; Gregg et al., 1980; Hicks et al., 2005; May et al., 1979; Van Overschelde, 2002; Ward et al., 2003; Watkins et al., 2000), separated by type of study list.

we seek to explain a phenomenon that involves high- and low-frequency words, it is prudent to examine the associations that develop between high- and low-frequency words, rather than words in general. Four conditional probabilities can therefore be examined: Given a high-frequency word was just recalled, what are the probabilities of recalling a high- (high|high) or a low- (low|high) frequency word next; and given a low-frequency word was just recalled, what are the probabilities of recalling a high- (high|low) and a low(low|low) frequency word next? These four probabilities can be examined within different list contexts (e.g., blocked, alternating, and random) to look for asymmetries in association that could help us understand list-based effects.

For example, imagine we observed a low-frequency recall advantage in the random list. If the item-to-item associations in this list were only sensitive to contiguity then the conditional probabilities of recalling a low- or high-frequency item after the current item should be roughly equal even if the probabilities of retrieving that current item did differ depending on its frequency. Thus, if the $P($ high $\mid$ high $)$ and $P($ high $\mid$ low $)$ were .40 and $P($ low $\mid$ high $)$ and $P($ low $\mid$ low $)$ were .60 , this would indicate that within-class frequency associations are not giving rise to the low-frequency advantage. Rather, it would simply suggest that low-frequency words were better recalled in general. However, if $P$ (high $\mid$ low $)$ was $.50, P($ low $\mid$ low $)$ was $.50, P$ (high $\mid$ high) was .40 , and $P$ (low $\mid$ high) was .60 , this would suggest that the low-frequency recall advantage occurred because low-frequency items are particularly easy to recall after a high-frequency item. Thus, we would have evidence that strong unidirectional high-low associations give rise to the low-frequency recall advantage.
The suggestion that some frequency-based associations may be stronger than others is not novel (e.g., Gillund \& Shiffrin, 1984). In fact, it seems intuitive that to produce a low-frequency recall advantage an asymmetry of frequency associations that biased participants toward the recall of low-frequency words would need to be observed. Thus, either $P($ low $\mid$ high $)$ or $P($ low $\mid$ low $)$ would need to be unusually strong. The second of these possibilities, that a low-frequency recall advantage could arise due to strong low-low associations, does not really make sense, because if this were the case, in blocked or pure lists lowfrequency words should also show a recall advantage, which they do not. Logically then, it should be the case that the $P$ (low $\mid$ high) should be particularly pronounced in lists that show a low-frequency recall advantage.

Theoretically, high-frequency words might be particularly prone to associate with low-frequency words as a result of the specificity of images brought to mind by the words. Nelson and McEvoy (2000) suggest that highfrequency words give rise to less specific images than do low-frequency words. Thus, when a high-frequency word is seen at study it may bring to mind a general image. If a low-frequency word is seen shortly thereafter, it may be relatively easy to somehow "fit" the more specific image of the low-frequency word to this less specific high-frequency image.

For example, imagine TABLE was seen at study. This highfrequency word would probably bring to mind a vague notion of a table. If NURSE, a low-frequency word, was seen shortly after, it should be relatively easy to link these two words, as the more concrete image of a nurse may bring to mind an operating table full of medical tools. If one then recalls TABLE at test, it might be relatively easy to recall NURSE next. Therefore, perhaps high-low pairings may be relatively easy associations for participants to make.

Our present goal is to examine the mixed-list paradox in detail to see if, when a low-frequency recall advantage does occur, it can be predicted by asymmetrical associations. To this end we examine different arrangements of study lists to see how associations between high- and low-frequency words might interact to produce the low-frequency recall advantage. If we are correct in our predictions, then lists that show a low-frequency advantage should also show an asymmetry of frequency associations that specifically manifests as unusually strong high-low associations.

\section{Present Experiment}

In the present experiment, we set out to investigate the associations and level of recall in blocked, alternating, and random lists. Four study conditions were used in our experiment: two blocked lists (i.e., high then low and low then high), an alternating condition (e.g., HLHLHLHL . . .), and a random condition (e.g., HHHLLHLL ....).

\section{METHOD}

\section{Participants}

Twenty-two students from the University of Toronto Scarborough participated in the experiment. All participants had normal or corrected-to-normal vision, and all were sufficiently proficient in English to complete the task without difficulty. 
Stimuli

Forty-eight high-frequency words and 48 low-frequency words were obtained from the MRC Linguistic Database. The highfrequency words had a mean KF frequency of $67.4(S D=20.6)$ and the low-frequency words had a mean KF of $1.5(S D=1.5)$. All words were between five and seven characters long.

\section{Procedure}

Each participant experienced four conditions of study-test recall. All began with instructions informing participants that a study list was soon to be presented to them, and that they would be responsible for remembering as many items as possible. In the high-low condition, 12 high-frequency words were followed by 12 low-frequency words. In low-high condition, 12 low-frequency words were followed by 12 high-frequency words. In the alternating condition, 12 high- and 12 low-frequency words were presented in an alternating order, starting with a high-frequency word. Finally, in the random condition, 12 high- and 12 low-frequency words were presented in a random order. The conditions were counterbalanced across participants such that all possible orders were used. ${ }^{1}$ During the study phase, each word was presented for $0.5 \mathrm{sec}$ with a 4.5 -sec interstimulus interval.

In the test phase, participants listed words, one at a time, as they recalled them. The experimenter checked the words off, noting the order. The test phase ended when the participant reported being unable to think of any more words. After completing a test phase, participants moved on to the study phase of the next condition and this continued until all four conditions had been completed.

\section{RESULTS AND DISCUSSION}

The results of the experiment were analyzed in three ways. First we consider the overall recall results, then we examine the conditional probabilities with respect to contiguity effects, and finally we examine the conditional probabilities reflecting the links between high- and lowfrequency words.

\section{Average Items Recalled}

The average number of correctly recalled high- and lowfrequency words can be seen in Table 1 broken down by condition. The mean number of intrusions was quite low and is reported only for completeness, as we had no interest in intrusions. A 2 (high vs. low frequency) $\times 4$ (condition) within-subjects ANOVA was conducted on the data. No main effect was found for condition $[F(3,63)<1]$ or word frequency $[F(1,21)<1]$. However, a significant interaction was observed $\left[F(3,63)=5.29, M S_{\mathrm{e}}=3.11, p<\right.$ $.01]$. To further examine this interaction, paired sample $t$ tests were performed.

Although there was a tendency to recall more highfrequency words when high- and low-frequency words were blocked, this result was not significant in either the high-low $[t(21)=1.0, p=.33]$ or the low-high $[t(21)=$ $1.4, p=.17]$ condition, when examined separately. However, when the two blocked conditions were combined, participants did recall more high-frequency words than low-frequency words $[t(21)=2.13, p<.05]$. In the alternating condition there was no difference in number of high- or low-frequency words recalled $[t(21)=0.11, p=$ $.92]$, but in the random condition, a low-frequency recall advantage was observed $[t(21)=3.13, p<.01]$.

The finding that high-frequency words are better recalled when high- and low-frequency words are blocked agrees with past research (e.g., May et al., 1979). Inter-
Table 1

Mean Number of Correctly Recalled Items and Intrusions, Separated by Block

\begin{tabular}{|c|c|c|c|c|c|c|}
\hline \multirow[b]{2}{*}{ Block } & \multicolumn{2}{|c|}{$\begin{array}{c}\text { High } \\
\text { Frequency }\end{array}$} & \multicolumn{2}{|c|}{$\begin{array}{c}\text { Low } \\
\text { Frequency }\end{array}$} & \multicolumn{2}{|c|}{ Intrusions } \\
\hline & $M$ & $S D$ & $M$ & $S D$ & $M$ & $S D$ \\
\hline High then low & 5.14 & 1.91 & 4.68 & 2.15 & 0.64 & 0.85 \\
\hline Low then high & 5.73 & 2.73 & 4.86 & 1.86 & 0.45 & 0.60 \\
\hline Alternating & 5.09 & 2.41 & 5.14 & 1.75 & 0.41 & 0.50 \\
\hline Random & 4.27 & 2.64 & 6.18 & 2.15 & 0.55 & 0.96 \\
\hline
\end{tabular}

estingly, a low-frequency recall advantage was observed only in the random condition, and not in the alternating condition. One possibility is that with only 22 participants we had insufficient power to detect a low-frequency recall advantage in the alternating condition but that an advantage does exist. We reject this possibility as a power analysis revealed that, given the observed effect size in the alternating condition, approximately 23,228 participants would be needed to have sufficient power to detect a difference. Thus, if an effect was present in the alternating condition, it was quite small. Given then, that we only observed a low-frequency recall advantage in the random condition, we predict that an asymmetry of frequency associability should be observed in the random condition but not in the alternating condition. Before examining the frequency associations, we first confirm that association by contiguity was occurring in our study.

\section{Conditional Response Probability of Distance}

Similar to Kahana (1996), conditional response probability plots were constructed for each condition of the experiment as presented in Figure 2. Each figure plots the probability of recalling items from study that were from one to five positions ahead or behind the just recalled item. A 10 (position of next item recalled, from -5 to +5 positions, excluding zero) $\times 4$ (condition) within-subjects ANOVA was conducted. ${ }^{2}$ There was no main effect of condition $\left[F(3,63)=1.36, M S_{\mathrm{e}}=0.01, p=.27\right]$ and no interaction between condition and position $[F(27,567)<1]$. There was however a main effect of position $[F(9,189)=$ $\left.34.23, M S_{\mathrm{e}}=0.01, p<.01\right]$, suggesting that participants were more likely to recall words in certain positions.

As can be seen in the plots, in all conditions there is a large probability of recalling an item that was one study position ahead of the currently recalled item. There is also a general tendency to recall items in the forward direction versus the backward direction, but also a relatively high probability of recalling an item one position previous from the current item. These patterns conform to those observed by Kahana (1996) in his evaluation of association by contiguity. ${ }^{3}$ Hence, it appears that association by contiguity was occurring similarly for all study conditions.

\section{Conditional Response Probability of Frequency}

Given that study items were associating by contiguity, we turn our attention to the question of whether frequency may have also affected interitem associations. To evaluate frequency associations, the four relevant conditional probabilities were calculated for each condition: $P($ high $\mid$ high), 


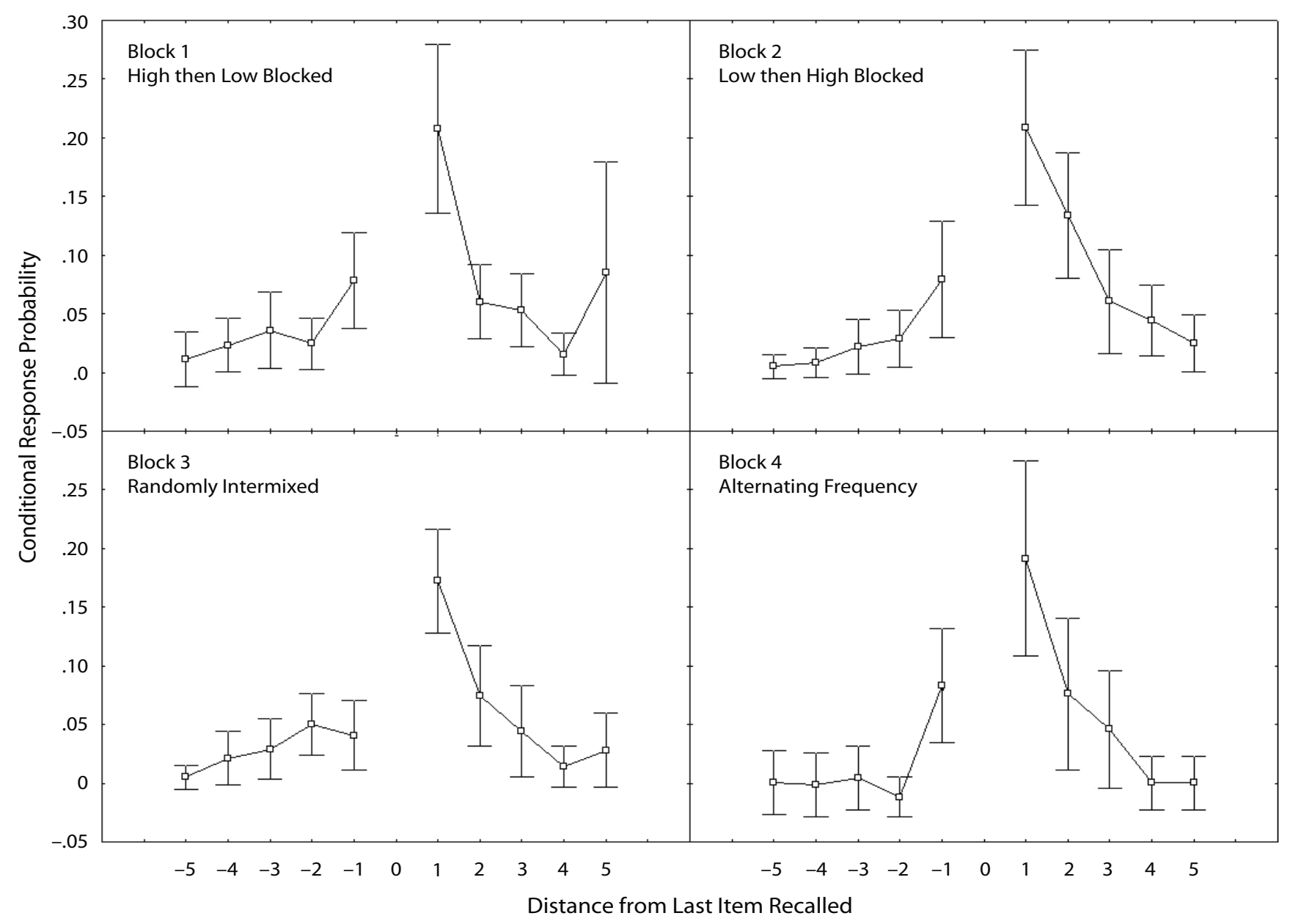

Figure 2. Mean conditional response probability plotted against distance from last item recalled, separated by block.

$P$ (low $\mid$ high), $P($ high $\mid$ low $)$, and $P$ (low $\mid$ low $)$. To be explicit, $P($ low $\mid$ high), for example, refers to the probability of recalling a low frequency study item next given that a high frequency study item was just recalled.

Each probability was calculated separately for each participant. For example, $P($ low $\mid$ high $)$ was calculated as the number of low-frequency words recalled following a high-frequency word, divided by the total number of highfrequency words recalled. These probabilities averaged across participants are presented in Table 2. Given that our interest was in specific differences between probabilities, paired sample $t$ tests were used to analyze these probabilities further.

In the high-low and low-high conditions, high- and lowfrequency words were blocked at study, and the predictions based on association were realized. That is, $P$ (high|high) was significantly greater than $P$ (high|low) for both of the pure-list conditions [both $t \mathrm{~s}(21)>4.00, p \mathrm{~s}<.01$ ]. Similarly, $P$ (low $\mid$ low $)$ was significantly greater than $P($ low $\mid$ high $)$ for both pure-list conditions [both $t \mathrm{~s}(21)=2.30, p \mathrm{~s}<.05$ ] Hence, when frequency is blocked at study, participants were most likely to stay within-frequency when recalling words, a result most likely due to association by contiguity.
In the alternating condition, association by contiguity would predict a high rate of between-frequency recalls. In accord with this prediction, $P$ (high $\mid$ low) was significantly greater than $P($ low $\mid$ low $)[t(21)=2.80, p<.05]$ and $P$ (low|high) was significantly greater than $P$ (high|high) $[t(21)=4.19, p<.01]$. These associations were largely symmetrical, ${ }^{4}$ however, which is consistent with the fact that no overall low-frequency advantage was observed in the alternating condition.

Finally, in the random condition, association by contiguity would predict an equal probability of recalling a high- or low-frequency word given either a high- or low-frequency word was currently recalled. However, given that a lowfrequency recall advantage was observed for the random list, we predicted that an asymmetry of associations should be apparent in the data. As expected, although $P$ (high|low) was not statistically different from $P$ (low $\mid$ low) $[t(21)=$ $0.14, p=.89], P($ low $\mid$ high $)$ was significantly greater than $P($ high $\mid$ high $)[t(21)=2.82, p<.05]$. Thus, as suggested by our logical consideration of study-list effects, it does appear that a low-frequency advantage in recall is due to a relative ease of recalling low-frequency items after a highfrequency item has just be recalled. 
Table 2

Mean Probability of Recalling a High- Versus a Low-Frequency Word Next, Given That a High- or Low-Frequency Word Has Already Been Recalled, As a Function of Block

\begin{tabular}{lcccccc}
\hline \multirow{2}{*}{ Block } & & \multicolumn{2}{c}{ High Next } & & \multicolumn{2}{c}{ Low Next } \\
\cline { 6 - 7 } High then low & Recalled & $M$ & $S D$ & & $M$ & $S D$ \\
\hline \multirow{2}{*}{ Low then high } & High & .63 & .22 & .33 & .19 \\
& Low & .36 & .29 & .64 & .29 \\
& High & .71 & .30 & .25 & .26 \\
Alternating & Low & .36 & .28 & .64 & .28 \\
& High & .29 & .24 & .71 & .24 \\
Random & Low & .64 & .23 & .36 & .23 \\
& High & .32 & .27 & .64 & .30 \\
& Low & .51 & .24 & .49 & .24 \\
\hline
\end{tabular}

\section{GENERAL DISCUSSION}

The random condition of our experiment was the only condition to show a low-frequency recall advantage. We hypothesized that if asymmetrical frequency associations can give rise to this effect, then they should be observed in lists where a low-frequency recall advantage is observed. Consistent with this hypothesis, in the random condition an asymmetry of frequency associations was observed which biased participants toward more recalls of lowfrequency words following high-frequency words. We can be assured that this increase in high-low associations was not simply due to the fact that more low-frequency words were recalled in general, as no similar increase in low-low associations was observed. In addition, in the other three conditions, where no low-frequency recall advantage was observed, no asymmetry of association existed.

One obvious question is if asymmetrical associations gave rise to the low-frequency recall advantage in the random condition, why did they not also appear and give rise to a low-frequency recall advantage in the other conditions, especially the alternating condition? That is, if the force driving a low-frequency advantage is an enhanced ability to link a low-frequency item to study when it follows a high-frequency item, one can understand why such associations would be difficult to form in the context of pure lists, but it would seem that alternating lists should provide the optimal context for creating such links given that every low-frequency item is preceded by a highfrequency item.

One relatively mundane possibility is that the difference observed would not be a systematic tendency and that, if similar experiments were conducted, alternating conditions would yield a pattern similar to that observed in our random condition. Given that very few studies in the past have included both a random and alternating condition, there currently is no basis for knowing how systematic this pattern of findings is.

A more interesting possibility is that this difference is systematic, and that there is some aspect of random lists that somehow exaggerates the recall of low-frequency items in the context of just recalled high-frequency items. In fact, there is some weak evidence suggesting that highlow associations were stronger (.71) than low-high associations (.64) in our alternating condition. However, a power analysis revealed that approximately 177 participants would be needed to demonstrate this difference to be statistically significant. Thus, the pattern is possibly present but weak.

At this point it is important to note that alternating lists are different from random lists in two related ways. First, random lists allow for runs of stimulus types, and it is entirely possible that the process responsible for binding a low-frequency item to a previously presented high-frequency item is especially potent if the item two items back was also high-frequency, something that never happens in an alternating list. Second, in alternating lists, the item that follows a low-frequency item is always high-frequency, a fact that is not true for random lists. Perhaps this $n+1$ item also benefits if the $n$ (i.e., lowfrequency item) is better recalled thanks to simple contiguity effects.

The larger theoretical point is that random lists should not be considered as equivalent to alternating lists, even though the probability of any given class of item appearing is $50 \%$ in both conditions when two classes of item are used. The systematicity of the alternating condition could well give rise to further associations that might work against a low-frequency advantage, and if we are to understand all the components that eventually give rise to such effects, we must consider random and alternating lists as different in potentially important ways.

In summary, the goal of this article was to demonstrate that asymmetrical frequency associations could be responsible for the low-frequency advantage sometimes observed in free recall. We have speculated that this asymmetry is caused by low-frequency items being more strongly associated with previously presented high-frequency items because of the nonspecific imagery produced by highfrequency items. Other alternative explanations are also possible. For example, strategy accounts (e.g., Watkins et al., 2000) or the order-encoding hypothesis (e.g., DeLosh \& McDaniel, 1996) could potentially account for our findings. Thus, while the exact cause of this asymmetry remains somewhat open, it does indeed appear that it is this asymmetry that underlies the enhanced recall of low frequency items.

Our work also suggests that any complete account of frequency effects in free recall may need to address three components: (1) What gives rise to the high-frequency advantage that is observed when pure lists are used? (2) How do interitem associations modulate this advantage when high- and low-frequency items are intermixed at study? (3) How are these interitem associations differentially affected in random versus alternating list contexts? Once these components are clearly understood, the mixed-list paradox will no longer be paradoxical, and our understanding of memory processes will be enhanced.

\section{AUTHOR NOTE}

J.D.O. is now at the University of Waterloo. This research was supported by a grant from the Natural Sciences and Engineering Research Council of Canada and the Premier's Research Excellence Award. We thank William Hockley and Marty Niewiadomski for discussions relevant to this work. Correspondence relating to this article may be sent to 
J. D. Ozubko, Department of Psychology, University of Waterloo, Waterloo, Ontario, N2L 3G1 Canada (e-mail: jdozubko@uwaterloo.ca).

\section{REFERENCES}

Brown, S. C., Conover, J. N., Flores, L. M., \& Goodman, K. M. (1991). Clustering and recall: Do high clusterers recall more than low clusterers because of clustering? Journal of Experimental Psychology: Learning, Memory, \& Cognition, 17, 710-721.

DeLosh, E. L., \& McDaniel, M. A. (1996). The role of order information in free recall: Application to the word-frequency effect. Journal of Experimental Psychology: Learning, Memory, \& Cognition, 22, 1136-1146.

Gillund, G., \& Shiffrin, R. M. (1984). A retrieval model for both recognition and recall. Psychological Review, 91, 1-67.

Gregg, V. H., Montgomery, D. C., \& Castano, D. (1980). Recall of common and uncommon words from pure and mixed lists. Journal of Verbal Learning \& Verbal Behaviour, 19, 240-245.

Hicks, J. L., Marsh, R. L., \& CoOK, G. I. (2005). An observation on the role of context variability in free recall. Journal of Experimental Psychology: Learning, Memory, \& Cognition, 31, 1160-1164.

Kahana, M. J. (1996). Associative retrieval processes in free recall. Memory \& Cognition, 24, 103-109.

May, R. B., Cuddy, L. J., \& Norton, J. M. (1979). Temporal contrast and the word frequency effect. Canadian Journal of Psychology, 33, 141-147.

Murdock, B. B. (1962). The serial position effect of free recall. Journal of Experimental Psychology, 64, 482-488.

Murdock, B. B., \& Metcalfe, J. A. (1978). Controlled rehearsal in single-trial free recall. Journal of Verbal Learning \& Verbal Behavior, 17, 309-324.

Murdock, B. B., \& ОKADA, R. (1970). Interresponse times in singletrial free recall. Journal of Verbal Learning \& Verbal Behavior, 86, 263-267.

Nelson, D. L., \& McEvoy, C. L. (2000). What is this thing called frequency? Memory \& Cognition, 28, 509-522.
VAn Overschelde, J. P. (2002). The influence of word frequency on recency effects in directed free recall. Journal of Experimental Psychology: Learning, Memory, \& Cognition, 28, 611-615.

Ward, G., Woodward, G., Stevens, A., \& Stinson, C. (2003). Using overt rehearsals to explain word frequency effects in free recall. Journal of Experimental Psychology: Learning, Memory, \& Cognition, 29, 186-210.

WatKins, M. J., LeCompte, D. C., \& Kim, K. (2000). Role of study strategy in recall of mixed lists of common and rare words. Journal of Experimental Psychology: Learning, Memory, \& Cognition, 26, 239-245.

\section{NOTES}

1. Note that because only 22 participants ended up being available for this experiment, two possible orders of blocks were not used.

2. Although homogeneity of variance could not be assumed in this ANOVA, the main methods of correcting for homogeneity of variance resulted in the same pattern of significance. As a result, the $p$ values reported here are those assuming homogeneity of variance.

3. More detailed analyses in which power functions were fit to each participant and separated by word frequency and condition were conducted, but none of the results were significant between groups or frequencies, confirming that association by contiguity was roughly equivalent across the conditions as well as between high- and low-frequency words.

4. The differences in probabilities may actually not be symmetrical in the alternating condition, however, since power analyses revealed approximately 177 participants would be needed to have sufficient power to test this difference. Thus, we can be assured that if the probabilities do deviate from symmetry, the deviation is small at best.

(Manuscript received July 24, 2006; revision accepted for publication December 12, 2006). 